\title{
ON THE STABILITY OF LAMINAR BOUNDARY LAYER FLOW*
}

\author{
BY SIN-I CHENG (Princeton Lniversity)
}

The stability of two dimensional small disturbances in laminar boundary layer flow has been extensively studied on the assumption that boundary layer flows are essentially parallel flows. The direct effect of the local pressure gradient on the calculation of the stability limits for incompressible boundary layer flow has been shown to be negligible under the approximation $(\alpha R)^{-1} \ll 1$, if the local velocity profile is used in the stability calculation. However, the assumption that the vertical velocity in the boundary layer flow plays negligible role has not received careful attention.

There is a qualitative argument that under the Prandtl boundary layer approximation, the variation of the mean flow properties in the $x$-direction within a few wave lengths of the disturbance is of the order of $R^{-1}$, which is negligibly small compared to unity. Therefore, the contributions of such terms due to $x$-gradients of pressure and temperature in the stability calculation can be neglected as higher order small quantities. This kind of argument should be investigated more closely so far as the vertical velocity component is concerned even though the vertical velocity component is a small quantity of the order of $R^{-1}$. The vertical velocity component produces a momentum transfer and an energy transfer across the boundary layer where both the disturbance quantities and the mean flow properties vary rapidly. The net effect of the transport processes may thus be much larger than the magnitude of the small agent that produces the transport processes. While the vertical velocity component of the flow and the gradients of the flow properties in the $x$-direction are small quantities of the same order the net effect of the former in the stability calculation will be shown to be much more important than that of the latter.

From the two dimensional forms of the equations of mass continuity, momentum and energy with variable viscosity and thermal conductivity coefficient and constant specific heats, the linearized system of partial differential equations for the amplitude functions of the periodic disturbances of the type exp [ia $(x-c t)]$ is obtained with two independent spatial variables $x$ and $y$. The fast varying, frequency dependent part of the disturbances as functions of $x$ is represented by the factor exp $(i \alpha x)$. The decay or growth of the amplitudes of the disturbances in the $x$-direction is slow and in the first approximation, the $\dot{x}$-gradients of the amplitude functions can be considered as independent of $x$. The system of disturbance equations can hence be considered as a set of ordinary differential equations for $f, \phi, \pi, r$ and $\theta$ with $y$ as the only independent variable. Thus, we have the following equations:

Continuity:

$$
\left(\phi^{\prime}+i f\right)+\frac{v}{\alpha} \frac{r^{\prime}}{\rho}=-i(w-c) \frac{r}{\rho}-\frac{\rho^{\prime}}{\rho} \phi-\frac{r}{\rho} \frac{v^{\prime}}{\alpha}-\frac{1}{\alpha \rho} \frac{\partial}{\partial x}(\rho f+w r)
$$

${ }^{*}$ Received December 15,1952 . This study was supported jointly by the Office of Naval Research (U.S.N.), Neetramies Branch, Mathematical Sciences Division, and by the Air Research and Development Command, U.S.A.F. under Contract No. N6-Ori-270, Task Order No. 6 Project Number NR061-049. The present paper is an abbreviation of Report No. 211, Aeronautical Engineering Department, Princeton Lniversity. 
First Momentum:

$$
\begin{aligned}
& \alpha \rho\left[i(w-c) f+w^{\prime} \phi\right]+\rho\left[f w_{x}+w f_{x}+v f^{\prime}\right]+\left[w w_{x}+v w^{\prime}\right] r=-\frac{1}{\gamma M T^{2}}\left(i \alpha \pi+\pi_{x}\right) \\
& +\frac{1}{R}\left[\left(\frac{4}{3} \mu_{1}+\frac{2}{3} \mu_{2}\right)\left(f_{x x}+2 i \alpha f_{x}-\alpha^{2} f\right)+\left(\frac{\mu_{1}}{3}+\frac{2}{3} \mu_{2}\right) \alpha\left(\phi_{x}^{\prime}+i \alpha \varphi^{\prime}\right)+\mu_{1} f^{\prime \prime}\right] \\
& +\frac{1}{R} \frac{d \mu_{1}}{d T}\left[\left(\frac{4}{3}+\frac{2}{3} \tau\right) T_{x}\left(f_{x}+i \alpha f\right)+\frac{2}{3}(\tau-1) T_{x} \alpha \phi^{\prime}+\alpha T^{\prime}\left(\phi_{x}+i \alpha \phi\right)+T^{\prime} f^{\prime}\right] \\
& \quad+\frac{1}{R} \frac{d \mu_{1}}{d T}\left[\left\{\left(\frac{4}{3}+\frac{2}{3} \tau\right) w_{x}+\frac{2}{3}(\tau-1) v^{\prime}\right\}\left(\theta_{x}+i \alpha \theta\right)+\left(v_{x}+w^{\prime}\right) \theta^{\prime}\right] \\
& \quad+\frac{1}{R} \frac{d \mu_{1}}{d T}\left[\left(\frac{4}{3}+\frac{2}{3} \tau\right) w_{x x}+w^{\prime \prime}+\left(\frac{1}{3}+\frac{2}{3} \tau\right) v_{x}^{\prime}\right] \theta
\end{aligned}
$$

Second Momentum:

$$
\begin{aligned}
& \alpha^{2} \rho[i(w-c) \phi]+\alpha \rho\left(v^{\prime} \phi+v \varphi^{\prime}\right)+\rho v_{x} f+\alpha \rho w \varphi_{x}+\left(w v_{x}+v v^{\prime}\right) r \\
& =\frac{\mu_{1}}{R}\left\{\left[f_{x}^{\prime}+i \alpha f^{\prime}+\alpha \varphi_{x x}+2 i \alpha^{2} \phi_{x}-\alpha^{3} \phi\right]\right. \\
& \left.\quad+\left(\frac{4}{3}+\frac{2}{3} \frac{\mu_{2}}{\mu_{1}}\right) \alpha \phi^{\prime \prime}+\frac{2}{3}\left(\frac{\mu_{2}}{\mu_{1}}-1\right)\left(f_{x}^{\prime}+i \alpha f^{\prime}\right)\right\} \\
& \quad+\frac{1}{R} \frac{d \mu_{1}}{d T}\left\{T_{x}\left(f^{\prime}+\alpha \phi_{x}+i \alpha^{2} \phi\right)+\left(\frac{4}{3}+\frac{2}{3} \tau\right) T^{\prime} \alpha \phi^{\prime}+\frac{2}{3}(\tau-1) T^{\prime}\left(f_{x}+i \alpha f\right)\right\} \\
& \quad+\frac{1}{R} \frac{d \mu_{1}}{d T}\left\{\left(v_{x}+w^{\prime}\right)\left(\theta_{x}+i \alpha \theta\right)+\left(\frac{4}{3}+\frac{2}{3} \tau\right) v^{\prime} \theta^{\prime}+\frac{2}{3}(\tau-1) w_{x} \theta^{\prime}\right\} \\
& \quad+\frac{1}{R} \frac{d \mu_{1}}{d T}\left\{v_{x x}+\left(\frac{4}{3}+\frac{2}{3} \tau\right) v^{\prime \prime}+\left(\frac{1}{3}+\frac{2}{3} \tau\right) w_{x}^{\prime}\right\} \theta-\frac{\pi^{\prime}}{\gamma M^{2}}
\end{aligned}
$$

\section{Energy:}

$$
\begin{aligned}
& \alpha \rho\left[i(w-c) \theta+T^{\prime} \phi\right]+\rho\left[w \theta_{x}+v \theta^{\prime}+T_{x} f\right]+\left(w T_{x}+v T^{\prime}\right) r \\
= & -(\gamma-1)\left[p\left(f_{x}+i \alpha f+\alpha \phi^{\prime}\right)+\left(w_{x}+v^{\prime}\right) \pi\right] \\
& +\mu_{1} \frac{\gamma(\gamma-1) M^{2}}{R}\left\{\left(\frac{4}{3}+\frac{2}{3} \frac{\mu_{2}}{\mu_{1}}\right) 2\left[w_{x}\left(f_{x}+i \alpha f\right)+\alpha v^{\prime} \phi^{\prime}\right]\right. \\
& \left.+\frac{4}{3}\left(\frac{\mu_{2}}{\mu_{1}}-1\right)\left[w_{x} \alpha \phi^{\prime}+v^{\prime}\left(f_{x}+i \alpha f\right)\right]+2\left(w^{\prime}+v_{x}\right)\left(f^{\prime}+\alpha \phi_{x}+i \alpha^{2} \phi\right)\right\} \\
& +\frac{d \mu_{1}}{d T} \frac{\gamma(\gamma-1)}{R} M^{2}\left[\left(\frac{4}{3}+\frac{2}{3} \tau\right)\left(w_{x}^{2}+v^{\prime 2}\right)+\frac{4}{3}(\tau-1) w_{x} v^{\prime}+\left(w^{\prime}+v_{x}\right)^{2}\right] \theta \\
& +\frac{\gamma \mu_{1}}{\sigma R}\left[\theta^{\prime \prime}+\theta_{x x}+2 i \alpha \theta_{x}-\alpha^{2} \theta\right] \\
& +\frac{d \mu_{1}}{d T} \frac{\gamma}{\sigma R}\left[T^{\prime \prime} \theta+T_{x x} \theta+2 T_{x}^{\prime}\left(\theta_{x}+i \alpha \theta\right)+2 T^{\prime} \theta^{\prime}\right]
\end{aligned}
$$


State:

$$
\frac{\pi}{p}=\frac{r}{\rho}+\frac{\theta}{T}
$$

where $\tau=\left[\left(d \mu_{2} / d T\right) /\left(d \mu_{1} / d T\right)\right]$, all other symbols are adopted from reference 1 .

For the convenience in reducing the equations the quantities $Z_{i}$ are defined in a manner slightly different from those in reference 1 and following the well known procedure, these variables $Z_{i}$ are expanded into series of $\epsilon=(\alpha R)^{1 / 3}$ as follows:

$$
\begin{array}{rlrl}
Z_{1} & = & f & =x_{1}^{(0)}+\epsilon x_{1}^{(1)}+\cdots \\
\epsilon Z_{2} & = & \epsilon f^{\prime} & =x_{2}^{(0)}+\epsilon x_{2}^{(1)}+\cdots \\
\epsilon^{-1} Z_{3} & = & \epsilon^{-1} \phi & =x_{3}^{(0)}+\epsilon x_{3}^{(1)}+\cdots \\
Z_{4} & =\quad \phi^{\prime} & =x_{4}^{(0)}+\epsilon x_{4}^{(1)}+\cdots \\
Z_{5} & =\quad \theta & =x_{5}^{(0)}+\epsilon x_{5}^{(1)}+\cdots \\
\epsilon Z_{6} & =\quad \epsilon \theta^{\prime} & =x_{8}^{(0)}+\epsilon x_{6}^{(1)}+\cdots \\
\epsilon^{-1} Z_{7} & =\epsilon^{-1} \frac{\pi}{M^{2}}=x_{7}^{(0)}+\epsilon x_{7}^{(1)}+\cdots \\
Z_{8} & =\frac{\pi^{\prime}}{M^{2}} &
\end{array}
$$

The mean flow velocity components $w$ and $v$, the mean density and temperature are also expanded into Taylor series about the critical point $y_{c}$ where $w\left(y_{c}\right)=c$. The expansion of $v$ is written as:

where

$$
\begin{aligned}
v & =\frac{1}{\rho} \int_{0}^{y} \frac{\partial}{\partial x}(\rho w) d y=\frac{1}{R} \cdot \frac{1}{\rho} \int_{0}^{y} \frac{\partial}{\partial \xi}(\rho w) d y \\
& =\alpha \epsilon^{3}\left[v_{0}+v_{1} \cdot \epsilon \eta+v_{2} \cdot(\epsilon \eta)^{2} / 2+\cdots\right]
\end{aligned}
$$

$$
\begin{aligned}
& v_{0}=\frac{1}{\rho_{c}}\left[\frac{\partial(\rho w)_{c}}{\partial \xi} y_{c}-\frac{\partial(\rho w)_{c}^{\prime}}{\partial \xi} y_{c}^{2} / 2+\cdots\right] \\
& v_{1}=\frac{1}{\rho_{c}}\left[\frac{\partial(\rho w)_{c}}{\partial \xi}-\frac{\rho_{c}^{\prime}}{\rho_{c}} v_{0}\right], \quad v_{2}=\cdots
\end{aligned}
$$

are of the order of unity for both compressible and incompressible flow. Substituting all these expansions into the disturbance equations and collecting terms of the same power of $\epsilon$, one obtains for the first approximation of the order of unity or $\epsilon^{0}$ :

$$
\left\{\begin{array}{l}
\frac{d x_{3}^{(0)}}{d \eta}+i x_{1}^{(0)}=0 \\
\frac{d^{2} x_{1}^{(0)}}{d \eta^{2}}-\frac{w_{c}^{\prime}}{\nu_{1 c}}\left(i \eta x_{1}^{(0)}+x_{3}^{(0)}\right)-\frac{i}{\gamma \mu_{1 c}} x_{7}^{(0)}=0 \\
\frac{d x_{7}^{(0)}}{d \eta}=0 \\
\frac{d^{2} x_{5}^{(0)}}{d \eta^{2}}-\frac{\sigma}{\nu_{1 c}}\left[i w_{c}^{\prime} \eta x_{5}^{(0)}+\left(T_{c}^{\prime}-\frac{\gamma-1}{\gamma} \frac{p_{c}^{\prime}}{\rho_{c}}\right) x_{3}^{(0)}\right]
\end{array}\right.
$$


These equations are identical with those given in reference 1 , and solutions can be obtained therefrom.

For the second approximation of the order of $\epsilon$ one obtains:

$$
\begin{aligned}
& \left\{\begin{array}{l}
\frac{d x_{3}^{(1)}}{d \eta}+i x_{1}^{(1)}=\frac{i w_{c}^{\prime}}{T_{c}} \eta x_{5}^{(0)}-\frac{\rho_{c}^{\prime}}{\rho_{c}} x_{3}^{(0)} \\
\frac{d^{2} x_{1}^{(1)}}{d \eta^{2}}-\frac{w_{c}^{\prime}}{\nu_{1 c}}\left(i \eta x_{1}^{(1)}+x_{3}^{(1)}\right)-\frac{i}{\gamma \mu_{1 c}} x_{7}^{(1)}
\end{array}\right. \\
& =\left\{\frac{w_{c}^{\prime \prime}}{\nu_{1 c}}+\frac{w_{c}^{\prime}}{\nu_{1 c}}\left[\frac{\rho_{c}^{\prime}}{\rho_{c}}-\left(\frac{d\left(\ln \mu_{1}\right)}{d T}\right)_{c} T_{c}^{\prime}\right]\right\} \eta\left(i \eta x_{1}^{(0)}+x_{3}^{(0)}\right)-i \frac{w_{c}^{\prime \prime}}{\nu_{1 c}} \frac{\eta^{2}}{2} x_{1}^{(0)} \\
& -\frac{i}{\gamma \mu_{1 c}}\left(\frac{d\left(\ln \mu_{1}\right)}{d T}\right)_{c} T_{c}^{\prime} \eta x_{7}^{(0)}-\left(\frac{d\left(\ln \mu_{1}\right)}{d T}\right)_{c}\left(w_{c}^{\prime} x_{b}^{(0)}+T_{c}^{\prime} x_{2}^{(0)}\right) \\
& +\frac{v_{0}}{\nu_{1 c}} x_{2}^{(0)} \\
& \frac{d x_{7}^{(1)}}{d \eta}=0 \\
& \frac{d^{2} x_{5}^{(1)}}{d \eta^{2}}-\frac{\sigma}{\nu_{1 c}}\left[i w_{c}^{\prime} \eta x_{5}^{(1)}+\left(T_{c}^{\prime}-\frac{\gamma-1}{\gamma} \frac{p_{c}^{\prime}}{\rho_{c}}\right) x_{3}^{(1)}\right] \\
& =\frac{\sigma}{\nu_{1 c}}\left\{\left[\frac{\rho_{c}^{\prime}}{\rho_{c}}-\left(\frac{d\left(\ln \mu_{1}\right)}{d T}\right)_{c} T_{c}^{\prime}\right]\left(i w_{c}^{\prime} \eta^{2} x_{5}^{(0)}+T_{c}^{\prime} \eta x_{3}^{(0)}\right)+i w_{c}^{\prime \prime} \frac{\eta^{2}}{2} x_{5}^{(0)}\right. \\
& \left.-\frac{\gamma-1}{\gamma}\left[\frac{p_{c}^{\prime \prime}}{\rho_{c}}-\left(\frac{d\left(\ln \mu_{1}\right)}{d T}\right)_{c} T_{c}^{\prime} \frac{p_{c}^{\prime}}{\rho_{c}}\right] \eta x_{3}^{(0)}+T_{c}^{\prime \prime} \eta x_{3}^{(0)}\right\} \\
& -\frac{\gamma-1}{\gamma} \frac{\sigma}{\mu_{1 c}} i w_{c}^{\prime} \eta M^{2} x_{7}^{(0)}-2 \sigma(\gamma-1) M^{2} w_{c}^{\prime} x_{2}^{(0)}-\left(\frac{d\left(\ln \mu_{1}\right)}{d T}\right)_{c} 2 T_{c}^{\prime} x_{6}^{(0)} \\
& +\frac{\sigma}{\nu_{1 c}} v_{0} x_{6}^{(0)}
\end{aligned}
$$

The homogeneous parts of equations (9) are the same as equations (8), and the inhomogeneous parts are known functions in terms of the solutions of equations (8) and mean flow properties. The second approximation can therefore be obtained by quadrature. It is observed that the vertical velocity component enters into both the momentum and the energy relations in the system of equations (9), while the $x$-gradients do not at this approximation. Terms involving $x$-gradients enter only at the next approximation of the order of $\epsilon^{2}$. Therefore, the effect of the vertical velocity component is more critical than the effect of the gradients in the $x$-direction in the stability calculation.

Since $x_{2 i}^{(0)}=\left(d x_{1 i}^{(0)} / d \eta\right)=0$ for $j=3,4,5$ and 6 , it is only the two viscous solutions $x_{11}^{(1)}$ and $x_{12}^{(1)}$ or $x_{31}^{(1)}$ and $x_{32}^{(1)}$ that are dependent on the vertical velocity component. $x_{6 j}^{(1)}$ are all $v$-dependent but they enter into the characteristic value problem as higher 
order small quantities even in the compressible case. The major effect of the vertical velocity component in the determination of the stability boundary is through $x_{31}^{(1)}$ and $x_{32}^{(1)}$.

Suppose we take all $x_{3 i}$ from the $\epsilon$ series and consistently take all six solutions to the order of $\epsilon$ i.e. $x_{3 i}^{(0)}+\epsilon x_{3 i}^{(1)}$ in the boundary value problem, the $v$-dependence of $x_{31}^{(1)}$ and $x_{32}^{(1)}$ indicates an inconsistency of the simplification of assuming the boundary layer flow as parallel flow. Fortunately, all the previous investigators are satisfied with the first approximation of the two viscous solutions $x_{31}^{(0)}$ and $x_{32}^{(0)}$ while they used $x_{33}^{(0)}+$ $\epsilon x_{33}^{(1)}$ and $x_{34}^{(0)}+\epsilon x_{34}^{(1)}$ or the two equivalent inviscid solutions or the inviscid solutions corrected for viscosity in the boundary value problems. Hence, the stability boundary as determined by any of these methods is independent of $v$ and their results are consistent with the assumption that boundary layer flows are parallel flows. Therefore, within the order of approximation attempted by previous investigators, the stability of the laminar boundary layer is determined only by the local flow properties for both the compressible and the incompressible flow.

The accuracy of the quantitative determination of the stability boundary as carried out in references 2,3 and 4, however, can not be improved merely by taking more terms in the $\epsilon$ series without including the effect of the vertical velocity component. It is unfortunate that, in some practical cases, the parameter $\epsilon$ may be only 0.1 near the minimum critical Reynolds number and as such the second approximation of the order of $\epsilon$ should better be considered for accurate determination of the stability limit and the initial amplification rate, in which cases, the effect of the vertical velocity component must be included.

In addition, at high Mach numbers, the vertical velocity component in the boundary layer is of the order of $M^{4} / R$, and may enter the stability problem of the laminar boundary layer even in the first approximation. The stability of the hypersonic laminar boundary layer, therefore, requires careful investigation.

\title{
REFERENCES
}

1. L. Lees and C. C. Lin, Investigation of the stability of the laminar boundary layer in a compressible fluid, N.A.C.A. T.N.1115 (1946).

2. L. Lees, The stability of laminar boundary layer in a compressible fluid, N.A.C.A. T.N. 1360 (1947).

3. J. Pretsch, Die Stabilität einer ebenen Laminarströmung bei Druckgefälle und Druckanstieg, Jahrbuch der Deutschen Luftfahrtforschung, 1, 58-75 (1941).

4. J. A. Laurmann, Stability of compressible laminar boundary layer with pressure gradient, College of Aeronautics, Cranfield, England, Report No. 48, (1951).

\section{STRESS-STRAIN RELATIONS, UNIQUENESS AND VARIATIONAL THEOREMS FOR ELASTIC-PLASTIC MATERIALS WITH A SINGULAR YIELD SURFACE*}

\author{
By W. T. KOITER (Technical University, Delft, Holland)
}

1. Plastic stress-strain relations. The state of stress at any point of a continuous medium is described by the stress tensor $\sigma_{i i}$ and may be represented by a point in ninedimensional stress space. It will be assumed that no yielding occurs if the stress point

*Received Dec. 22, 1952. 